\title{
Wear Identification of Vibration Drilling Bit Based on Improved LMD and BP Neural Network
}

\author{
Yaochen Shi $\mathbb{D}^{1}{ }^{1}$ Hongyan Liu, ${ }^{2}$ Xuechen Zhang, ${ }^{3}$ Qinghua Li, ${ }^{1}$ and Xiaocheng Guo ${ }^{2}$ \\ ${ }^{1}$ College of Mechanical and Vehicle Engineering, Changchun University, Changchun, China \\ ${ }^{2}$ College of Mechanical Engineering, Changchun University of Science and Technology, Changchun, China \\ ${ }^{3}$ College of Mechanical Engineering, Changchun Guanghua University, Changchun, China
}

Correspondence should be addressed to Yaochen Shi; 250793721@qq.com

Received 23 May 2020; Accepted 4 July 2020; Published 14 August 2020

Academic Editor: Xiaoliang Jin

Copyright ( $\odot 2020$ Yaochen Shi et al. This is an open access article distributed under the Creative Commons Attribution License, which permits unrestricted use, distribution, and reproduction in any medium, provided the original work is properly cited.

In view of the low accuracy of single signal monitoring for the wear state of vibration drilling bit, a multisignal acquisition system for the wear state of ultrasonic axial vibration drilling bit is built to collect the drilling force, vibration, and acoustic emission signals under three different wear states. The drilling force, vibration and acoustic emission signals of the bit in the drilling process are processed by using wavelet decomposition technology, and the signals are extracted from the wear state of the bit, The wavelet energy coefficient with high state correlation is used as the feature parameter to identify the bit wear state. The feature parameter is trained by the combination of noise assisted LMD method and BP neural network. The experiment of single signal and multisignal fusion monitoring bit wear state is carried out, and the neural network structure is optimized according to the error. The results show that the accuracy of monitoring bit wear with a single signal of drilling force is $83.3 \%$, the accuracy of monitoring bit wear with a single signal of vibration is $91.6 \%$, the accuracy of monitoring bit wear with a single signal of acoustic emission is $91.6 \%$, and the accuracy of monitoring bit wear with multisignal fusion is $95.8 \%$; when the number of network layer is 4 , the vibration is monitored with the fusion of force signal, acoustic emission signal, and vibration signal The accuracy of the state of drilling tool is up to $100 \%$. The structure model of neural network is optimized reasonably to improve the recognition rate of bit wear in vibration drilling.

\section{Introduction}

Ultrasonic vibration drilling has many advantages, such as good chip breaking and chip removal performance, smooth hole wall surface, etc., which is widely used in deep hole drilling [1]. In drilling, the state of the tool is one of the main factors affecting the machining efficiency and workpiece quality [2]. Drilling is carried out in a semiclosed state. During the drilling process, it is impossible to directly observe and monitor the wear state of the tool. It is mainly through the operator's experience to judge the wear degree of the tool and determine the tool change strategy. However, this method has poor reliability and affects the machining efficiency. With the development of machine tools, in the direction of automation, integration, and unmanned development, the research of intelligent tool monitoring technology is particularly important [3]. Therefore, experts at home and abroad have carried out many related researches on it, such as taking acoustic emission, vibration, power, force, and current signals as monitoring signals, collecting features related to tool status, and indirectly monitoring tool status [4-8]. Since the data collected by sensors are large and the physical dimensions of signals collected by different sensors are different, simultaneous interpreting of tool wear state is adopted. In 2015, Azmi verified that the feed force in the milling process has awesome prediction for tool wear state and established a milling cutter condition monitoring system based on feed force monitoring signal, which has awesome milling cutter status recognition [9]. In 2017, Gao established a BTA bit wear condition monitoring system with cutting power as the monitoring signal. The system uses RBF neural network to 


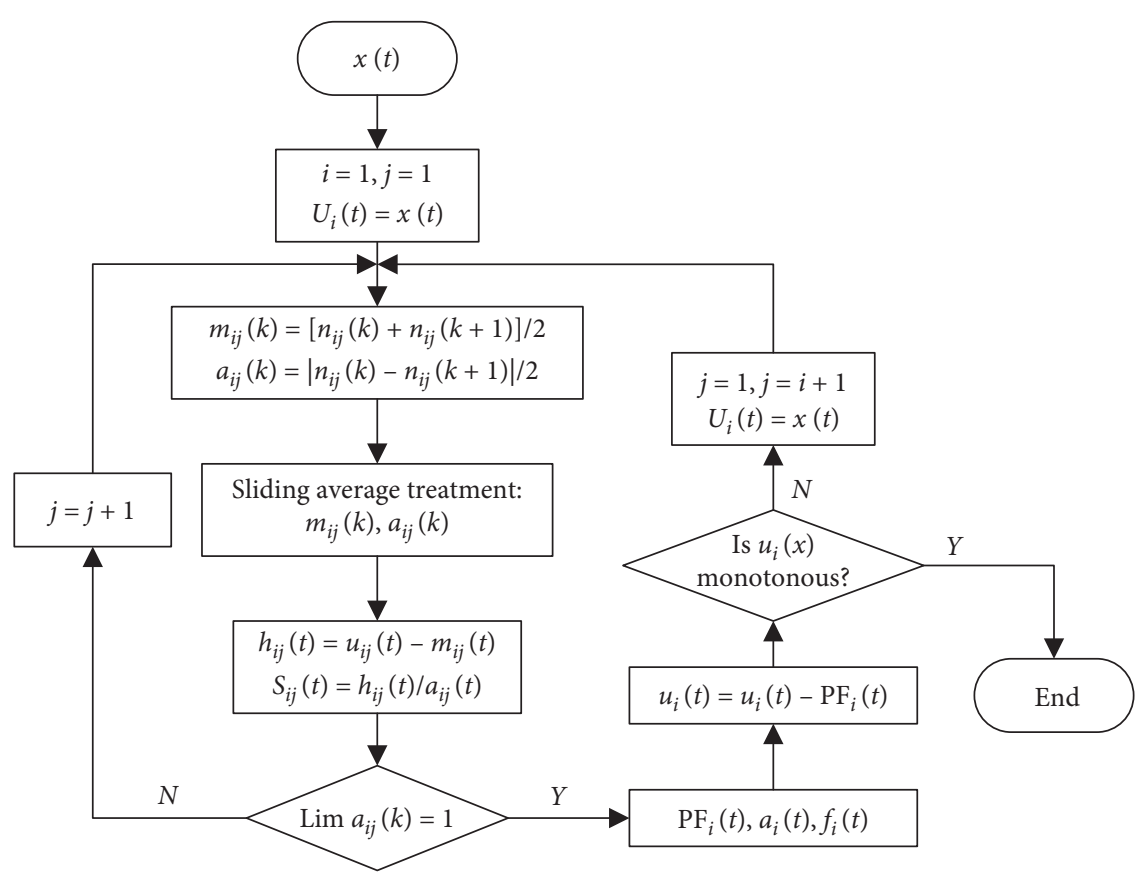

FIgURE 1: LMD decomposition flow chart.

identify the tool wear condition, and the recognition accuracy can reach $90 \%$ [10].

Using a single signal to monitor the status of cutting tools has the problem of low recognition rate. Therefore, in this paper, a multisignal acquisition system for the wear state of the axial vibration drilling bit is built with the drilling force, vibration, and acoustic emission signals as the monitoring signals. The wear state monitoring test of the 7075 aluminum plate is carried out on the $40 \mathrm{kHz}$ ultrasonic vibration drilling device. The drilling force, vibration and acoustic emission signals collected under different wear conditions are processed by wavelet decomposition, and the wavelet energy coefficient with high correlation with the bit wear condition is obtained as the characteristic parameter, which is input into the BP neural network model to identify the bit wear condition, and the recognition result is compared with that based on a single signal to identify the bit wear condition. The results of damage state discrimination are compared and analyzed.

\section{LMD and Its Improvement}

2.1. The Brief Introduction of LMD. Local mean decomposition (LMD) is proposed by Smith. It can decompose the original complex signal with multiple components into the sum of PF components of a series of single frequency signals, which has good adaptability and can improve the end effect of empirical mode decomposition [11]. For the given original signal $x(t)$, the decomposition flow is shown in Figure 1. In the figure, the mean value function is $m_{11}(t)$; the envelope function is $a_{11}(t)$; the pure frequency modulation signal is $S_{1 n}(t)$; the envelope signal is $a_{11}(t)$.

2.2. Noise-Aided Modified LMD Method. Since the traditional LMD method has the defect of modal aliasing, it is difficult to extract the accurate eigenvector of the false pf component. To solve this problem, the local mean decomposition is introduced, and the monitoring signal with white noise superimposed by LMD decomposition is used, and different white noise signals with a mean value of 0 are taken each time. Then, the mean value of the decomposition results is calculated many times, the noise signal is eliminated, the signal-to-noise ratio is improved, and the modal aliasing image is reduced. The specific process is shown in Figure 2.

\section{Test Conditions and Feature Extraction}

3.1. Test Conditions. In the test, NX1204-50 high-precision numerical control microhole bench drill, axial piezoelectric vibration system, and three-way cutting force measuring instrument (range in $x, y$ direction: $-5-5 \mathrm{kN}$; range in $z$ direction: $-5-10 \mathrm{kN}$; Sensitivity in $x, y$ direction: $-7.5 \mathrm{pC} / \mathrm{N}$; sensitivity in $z$ direction: $-3.7 \mathrm{pC} / \mathrm{N}$ ), SR150 $\mathrm{m}$ acoustic emission sensor (working frequency: $80-1100 \mathrm{kHz}$; sensitivity: >65 HB), CA-YD-152A three-way acceleration sensor (measuring range: $20 \mathrm{mV} / \mathrm{g}$; frequency range: $0.5-4 \mathrm{kHz}$ ), signal amplifier and data acquisition card matched with the sensor jointly build multisignal of wear state of axial vibration drilling bit. The number acquisition system is shown in Figure 3.

The workpiece is fixed on the piezoelectric sensor and connected with the workbench through the fixture, the acceleration sensor is close to the end face of the workpiece, and the AE sensor is installed on the side of the workpiece close to the acceleration sensor. The drill used in the test is YG8 tungsten steel fixed shank microdrill with a diameter of $1 \mathrm{~mm}$. The workpiece is a 7075 aluminum plate with a length of $90 \mathrm{~mm}$, a width of $60 \mathrm{~mm}$, and a thickness of $5 \mathrm{~mm}$. In the test, the selected parameters are shown in Table 1 . 


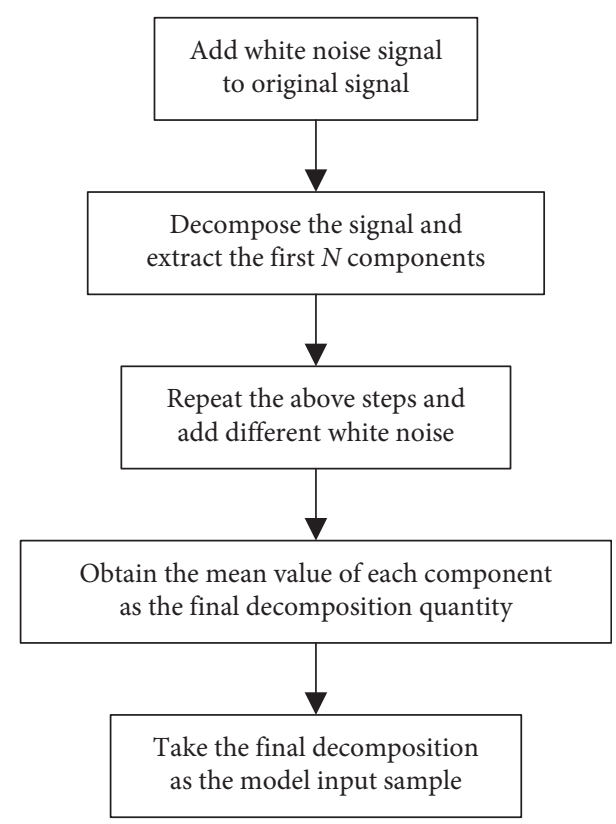

FIGURE 2: Flow chart of the LMD signal processing method based on noise assistance.

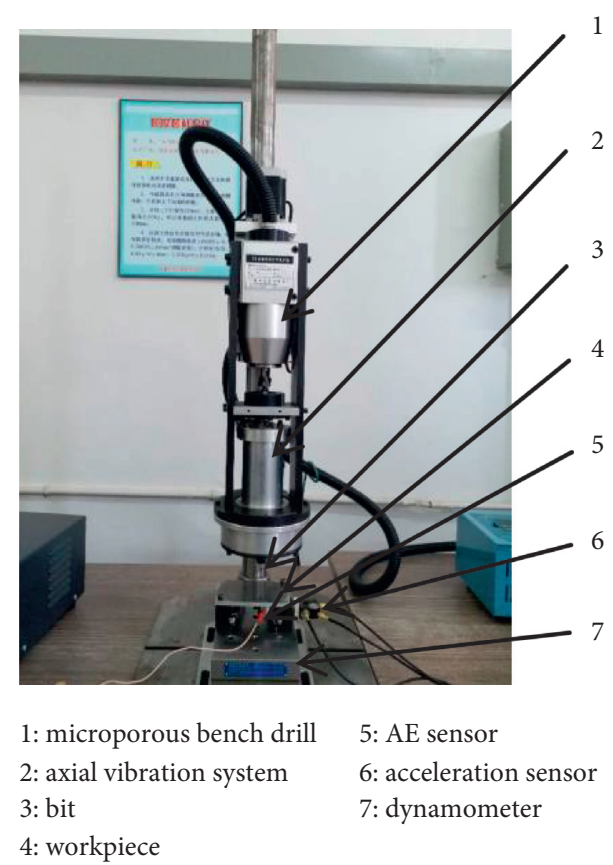

Figure 3: Physical diagram of a multisignal acquisition system for bit wear.

The wear monitoring test of ultrasonic vibration drilling bit was carried out on a 7075 aluminum plate to collect and process the drilling force, vibration, and acoustic emission signals of the bit under different wear degrees. In the test, every two holes are drilled, the wear of the drill bit is measured and recorded by a Leica dvm 2500 3D ultradepth of field electron microscope until the drill bit is scrapped. According to the processing conditions of the drill bit in the test, the wear state is divided, as shown in Table 2.
TABLE 1: Main parameters in drilling.

\begin{tabular}{lc}
\hline Spindle speed & $3000 \mathrm{r} / \mathrm{min}$ \\
\hline Feed rate & $2 \mu \mathrm{m} / \mathrm{r}$ \\
Vibration frequency & $40 \mathrm{kHz}$ \\
Amplitude & $13 \mu \mathrm{m}$ \\
\hline
\end{tabular}

TABLE 2: Classification of bit wear.

\begin{tabular}{lccc}
\hline Tool condition & Initial wear & Normal wear & Severe wear \\
\hline$V_{B} / \mathrm{mm}$ & $0-0.05$ & $0.05-0.15$ & $\geq 0.15$ \\
\hline
\end{tabular}

3.2. Feature Extraction. In the drilling process, the collected drilling force, acoustic emission, and vibration signals will generate random fluctuations, which belongs to nonstationary random signals [12-14]. For nonstationary random signals, the time-domain analysis method is used to judge the bit wear state with certain error, and the frequencydomain analysis method cannot be used to get the law of frequency change with time. Wavelet decomposition has the characteristics of multiresolution analysis and has the ability to characterize signal characteristics in time and frequency domain [15]. Therefore, in this paper, the wavelet decomposition method is used to analyze and process the collected signal in the drilling area and extract the feature vector related to the bit wear state.

3.2.1. Feature Extraction of Drilling Force Signal. The sampling frequency of the drilling force signal acquisition system is $1 \mathrm{kHz}$ and the sampling length is $5 \times 10^{4}$. In consideration of the actual vibration drilling process, affected by the processing conditions, the monitoring signal collected by the sensor is often mixed with a lot of noise, which reduces the effectiveness of the signal. Through the test, it is found that the $\mathrm{db} 4$ wavelet basis function can reduce noise interference and improve the signal-to-noise ratio. Therefore, in this paper, $\mathrm{db} 4$ wavelet basis function is used to conduct 5-layer wavelet decomposition for the middrill region of axial drilling force signal collected under three wear states, and the sampling points collected are $5 \times 10^{4}$. After wavelet decomposition, the resolution of signal frequency is $31.25 \mathrm{~Hz}$. The frequency range corresponding to the frequency band of 6 wavelet energy coefficients is shown in Table 3. The result of wavelet decomposition of drilling force signal in severe wear stage of vibration drilling bit based on improved LMD is shown in Figure 4.

Figure 5 shows the energy distribution of the drilling force signal in different frequency bands.

It can be seen from Figure 5 that the energy of the drilling force signal after wavelet decomposition is mainly distributed in A5 and D4 frequency bands. With the increase of bit wear degree, the energy coefficient of axial drilling force signal in high frequency band increases gradually, among which the energy coefficient of bit with different wear degree in D4 band changes most clearly, while the energy coefficient of A5 in low frequency band decreases gradually, which has a good corresponding relationship with the change of bit wear state. Therefore, D4 and A5 after wavelet decomposition of 
TABLE 3: Five-Layer wavelet decomposition frequency band of axial drilling force signal.

\begin{tabular}{lcccccc}
\hline Serial number & D1 & D2 & D3 & D4 & D5 & A5 \\
\hline Frequency band $(\mathrm{Hz})$ & $500 \sim 250$ & $250 \sim 125$ & $125 \sim 62.5$ & $62.5 \sim 31.25$ & $31.25 \sim 15.625$ & $15.625 \sim 0$ \\
\hline
\end{tabular}
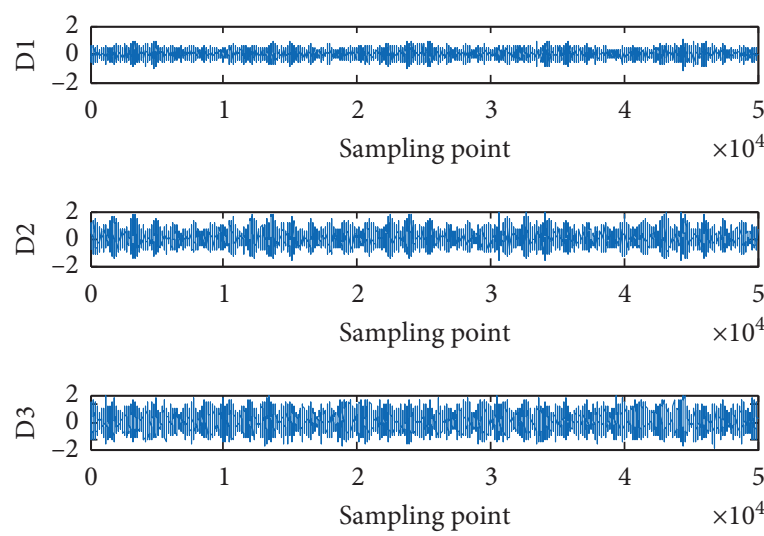
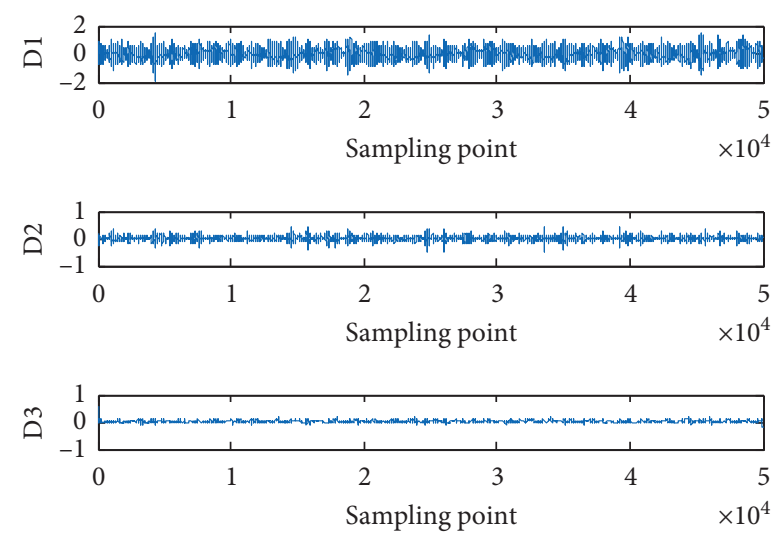

FIGURE 4: Five-layer wavelet decomposition of drilling force signal.
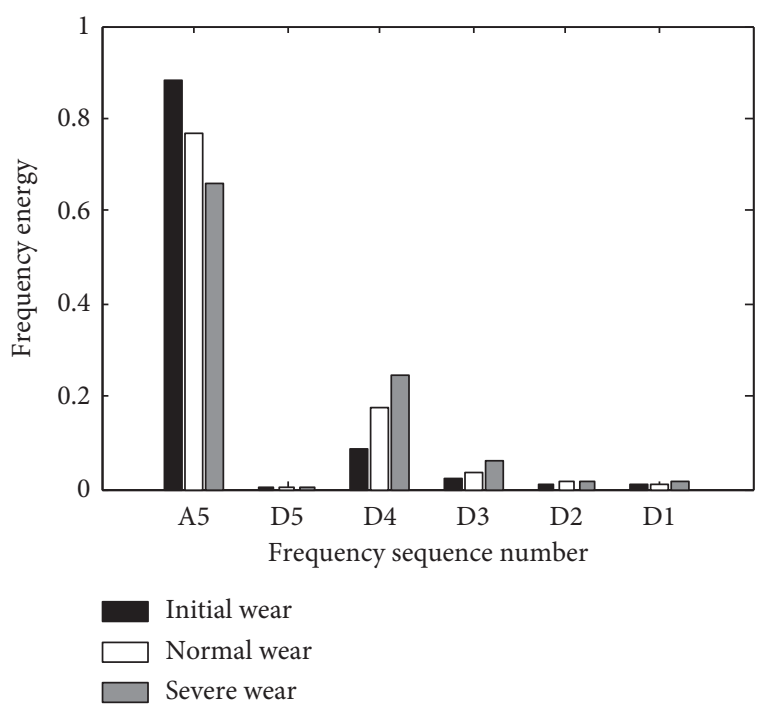

FIGURE 5: Energy variation curve of drilling force signal in different frequency bands.

axial drilling force signal are selected. The wavelet energy coefficient of the frequency band is the characteristic parameter of bit wear condition monitoring.

3.2.2. Feature Extraction of Vibration Signal. The sampling frequency of the vibration signal acquisition system is $500 \mathrm{kHz}$, and the sampling point is $5 \times 10^{4}$. To reduce the impact of noise in the processing environment, $\mathrm{db} 4$ wavelet basis function is selected to decompose the $z$-direction vibration signal in the drilling area into the collected three wear states by five layers of wavelet, and the distribution of wavelet energy coefficient of each frequency band of the bit in different wear states is shown in Figure 6 and the resolution of signal frequency after wavelet decomposition is $15.625 \mathrm{kHz}$.
It can be seen from Figure 6 that, with the increase of bit wear, the D4 and D1 band energy of vibration signal increase gradually, while the A5 band energy decreases gradually, and the other band energy does not change significantly. Therefore, this paper selects the wavelet energy coefficients of D4, D1, and A5 frequency bands extracted after wavelet decomposition of vibration signals as the characteristic parameters of bit wear condition monitoring.

3.2.3. Feature Extraction of AE Signal. The acoustic emission signal of metal material is a high frequency signal [16] whose frequency range is generally between $50 \mathrm{kHz}$ and $1 \mathrm{MHz}$. The sampling frequency of the AE signal acquisition system is set as $500 \mathrm{kHz}$ and the sampling point is $5 \times 10^{4}$. In this paper, the sym6 wavelet basis function, which can effectively improve the signal-to-noise ratio, is used to decompose the area of AE signal in drilling under different wear conditions into five layers. According to the wavelet energy coefficient analysis method, the distribution of the wavelet energy coefficient in each frequency band of drill bit under different wear conditions is obtained. The whole frequency domain is divided into six frequency bands, from low frequency to high frequency A5, D5, D4, D3, For D2, and D1; the resolution of signal frequency after wavelet decomposition is $15.625 \mathrm{kHz}$, and the AE signal energy comparison of drill bit under different wearing conditions is shown in Figure 7.

As can be seen from Figure 7, the energy of the AE signal after wavelet decomposition is mainly concentrated in D4, D3, D2, and D1 frequency bands. With the increase of bit, the energy of D1 and D3 band increases, while the energy of the D2 band decreases, while the energy of other bands does not change significantly. The greater the influence of wear state on the energy fluctuation in the same frequency band, the better the correlation between the frequency band and bit wear state. Therefore, the wavelet energy coefficients of D1, D2, and D3 bands extracted from AE signals after 


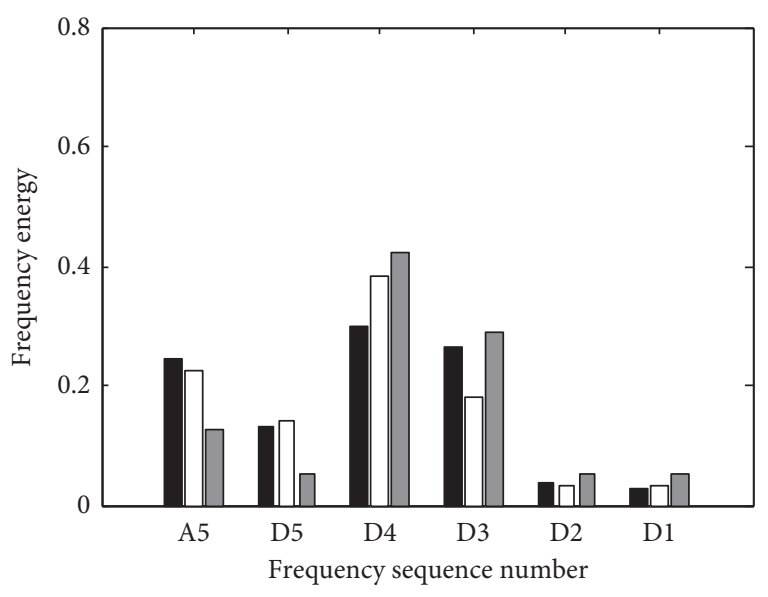

Initial wear

$\square$ Normal wear

$\square$ Severe wear

FIGURE 6: Energy variation diagram of vibration signal in different frequency bands.

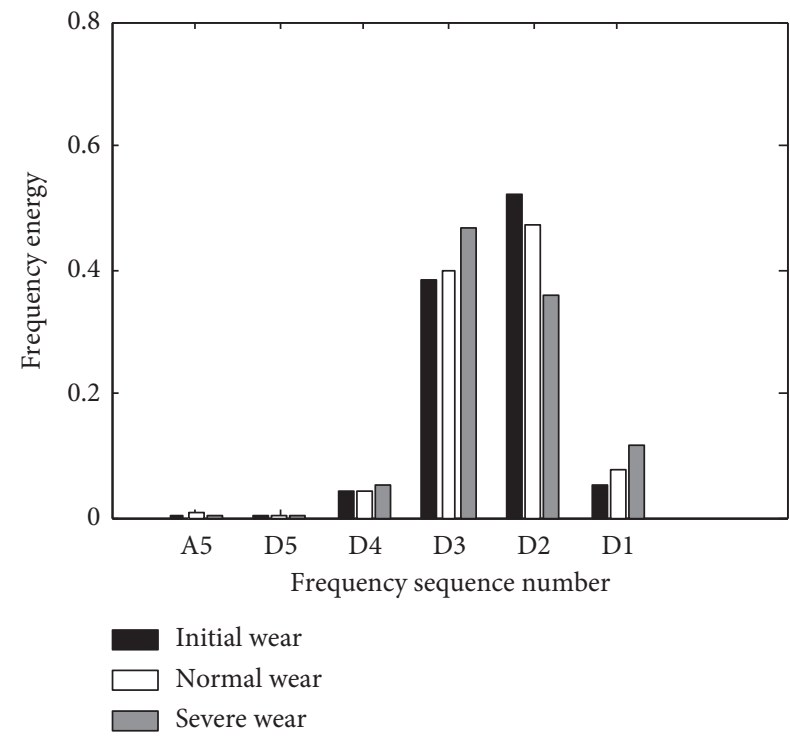

FIGURE 7: Energy variation diagram of the AE signal in different frequency bands.

wavelet decomposition are selected as the characteristic parameters of bit wear condition monitoring.

\section{Recognition Model of Bit Wear State}

Through BP neural network, the nonlinear mapping relationship between the bit wear state and signal eigenvector can be constructed to judge the bit wear state [17]. Therefore, this paper uses BP neural network technology to fuse the feature bands extracted from the wavelet decomposition of drilling force, $\mathrm{AE}$, and vibration signals, to realize the recognition of tool wear state in ultrasonic vibration drilling.

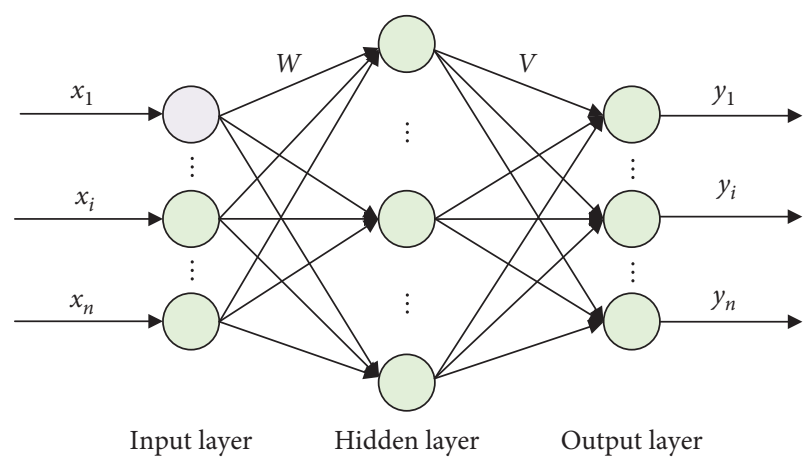

Figure 8: The structure diagram of the BP neural network with three layers.

TABLE 4: Corresponding relationship between output value and tool state.

\begin{tabular}{lccc}
\hline Tool condition & Initial wear & Normal wear & Severe wear \\
\hline Output value & {$\left[\begin{array}{lll}1 & 0 & 0\end{array}\right]$} & {$\left[\begin{array}{lll}0 & 1 & 0\end{array}\right]$} & {$\left[\begin{array}{lll}0 & 0 & 1\end{array}\right]$} \\
\hline
\end{tabular}

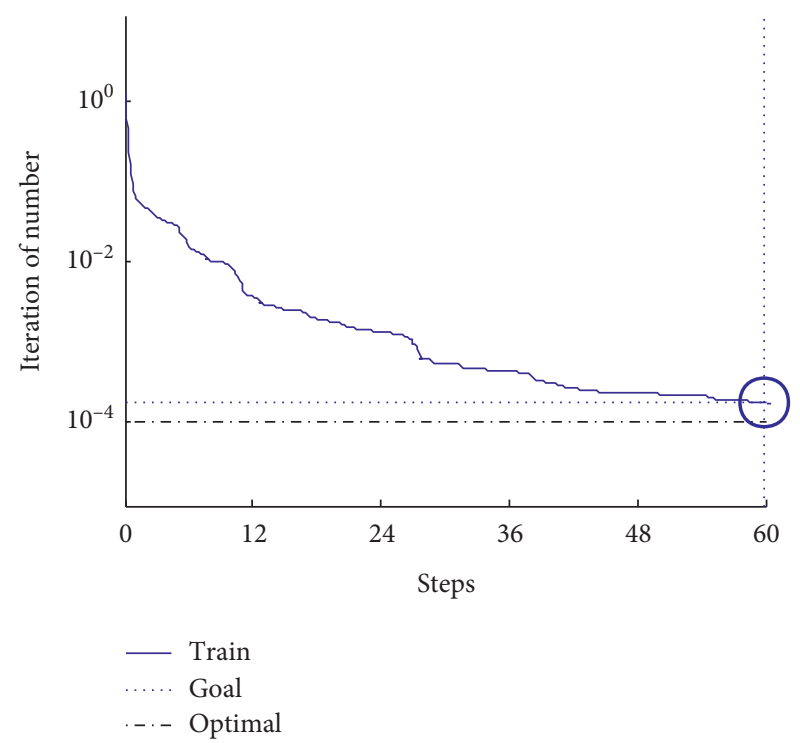

Figure 9: Training error curve of BP neural network.

\subsection{Structural Parameter Design of Neural Network}

4.1.1. Determination of Network Layers. For the BP neural network, the more the number of hidden layers is, the more unreliable the error is transferred from the output layer to the input layer in the reverse direction, and the more inaccurate correction of the weight value will lead to the performance degradation of BP neural network. According to Kolmogorov's theorem, a fully learned 3-layer BP neural network can approach any function [18]. Therefore, this paper selects three layers of BP neural network to identify the wear state of the drill bit.

4.1.2. Determination of the Number of Neurons. The number of neurons in the input layer of the BP neural network is determined by the number of eigenvectors that make up the 
TABle 5: Comparison of recognition results.

\begin{tabular}{|c|c|c|c|c|c|}
\hline Number & $\begin{array}{l}\text { Tool wear } \\
\text { condition }\end{array}$ & $\begin{array}{l}\text { Actual output (three signal } \\
\text { fusion) }\end{array}$ & $\begin{array}{l}\text { Actual output (force } \\
\text { signal) }\end{array}$ & Actual output (AE signal) & $\begin{array}{c}\text { Actual output (vibration } \\
\text { signal) }\end{array}$ \\
\hline 1 & Intial & {$\left[\begin{array}{llll}0.9461 & 0.0126 & 0.0109\end{array}\right]$} & {$\left[\begin{array}{llll}0.9054 & 0.0011 & 0.0114\end{array}\right]$} & {$\left[\begin{array}{lll}0.9920 & 0.0335 & 0.0046\end{array}\right]$} & {$\left[\begin{array}{llll}0.9756 & 0.0012 & 0.0193\end{array}\right]$} \\
\hline 2 & Initial & {$\left[\begin{array}{lll}0.9233 & 0.0052 & 0.0351\end{array}\right]$} & {$\left[\begin{array}{llll}0.9745 & 0.0301 & 0.0143\end{array}\right]$} & {$\left[\begin{array}{lll}0.9455 & 0.0195 & 0.0211\end{array}\right]$} & {$\left[\begin{array}{lll}0.9533 & 0.0127 & 0.0279\end{array}\right]$} \\
\hline 3 & Initial & {$\left[\begin{array}{lll}0.9665 & 0.0271 & 0.0264\end{array}\right]$} & {$\left[\begin{array}{lll}0.8910 & 0.0241 & 0.0108\end{array}\right]$} & {$\left[\begin{array}{lll}0.8961 & 0.0359 & 0.0152\end{array}\right]$} & {$\left[\begin{array}{lll}0.9873 & 0.0299 & 0.0075\end{array}\right]$} \\
\hline 4 & Initial & {$\left[\begin{array}{lll}0.9834 & 0.0074 & 0.0142\end{array}\right]$} & {$\left[\begin{array}{lll}0.9471 & 0.0099 & 0.0241\end{array}\right]$} & {$\left[\begin{array}{lll}0.9945 & 0.0217 & 0.0046\end{array}\right]$} & {$\left[\begin{array}{lll}0.9681 & 0.0018 & 0.0179\end{array}\right]$} \\
\hline 5 & Initial & {$\left[\begin{array}{lll}0.9533 & 0.0341 & 0.0326\end{array}\right]$} & {$\left[\begin{array}{llll}0.9466 & 0.0150 & 0.0223\end{array}\right]$} & {$\left[\begin{array}{lll}0.9586 & 0.0310 & 0.0272\end{array}\right]$} & {$\left[\begin{array}{lll}0.9550 & 0.0026 & 0.0501\end{array}\right]$} \\
\hline 6 & Initial & {$\left[\begin{array}{lll}0.8703 & 0.0052 & 0.0115\end{array}\right]$} & {$\left[\begin{array}{lll}0.0161 & 0.9403 & 0.0544\end{array}\right]$} & {$\left[\begin{array}{lll}0.9985 & 0.0803 & 0.0139\end{array}\right]$} & {$\left[\begin{array}{lll}0.9383 & 0.0118 & 0.0043\end{array}\right]$} \\
\hline 7 & Initial & {$\left[\begin{array}{lll}0.9115 & 0.0271 & 0.0184\end{array}\right]$} & {$\left[\begin{array}{lll}0.9722 & 0.0183 & 0.0124\end{array}\right]$} & {$\left[\begin{array}{lll}0.8268 & 0.0331 & 0.0054\end{array}\right]$} & {$\left[\begin{array}{lll}0.8754 & 0.0074 & 0.0211\end{array}\right]$} \\
\hline 8 & Initial & {$\left[\begin{array}{lll}0.9734 & 0.0062 & 0.0121\end{array}\right]$} & {$\left[\begin{array}{lll}0.9101 & 0.0138 & 0.0079\end{array}\right]$} & {$\left[\begin{array}{lll}0.9677 & 0.0356 & 0.0038\end{array}\right]$} & {$\left[\begin{array}{lll}0.9314 & 0.0138 & 0.0088\end{array}\right]$} \\
\hline 9 & Normal & {$\left[\begin{array}{llll}0.0193 & 0.9601 & 0.0009\end{array}\right]$} & {$\left[\begin{array}{llll}0.0319 & 0.9155 & 0.0032\end{array}\right]$} & {$\left[\begin{array}{lll}0.0025 & 0.9291 & 0.0166\end{array}\right]$} & {$\left[\begin{array}{lll}0.0323 & 0.9051 & 0.0429\end{array}\right]$} \\
\hline 10 & Normal & {$\left[\begin{array}{lll}0.0553 & 0.9305 & 0.0017\end{array}\right]$} & {$\left[\begin{array}{llll}0.0166 & 0.9775 & 0.0207\end{array}\right]$} & {$\left[\begin{array}{lll}0.8696 & 0.0372 & 0.0081\end{array}\right]$} & {$\left[\begin{array}{lll}0.0037 & 0.9457 & 0.0009\end{array}\right]$} \\
\hline 11 & Normal & {$\left[\begin{array}{lll}0.0613 & 0.8201 & 0.0126\end{array}\right]$} & {$\left[\begin{array}{lll}0.0960 & 0.0531 & 0.9138\end{array}\right]$} & {$\left[\begin{array}{lll}0.0033 & 0.9435 & 0.0355\end{array}\right]$} & {$\left[\begin{array}{lll}0.0316 & 0.0071 & 0.8788\end{array}\right]$} \\
\hline 12 & Normal & {$\left[\begin{array}{lll}0.0159 & 0.8776 & 0.0284\end{array}\right]$} & {$\left[\begin{array}{llll}0.0088 & 0.9236 & 0.0431\end{array}\right]$} & {$\left[\begin{array}{lll}0.0188 & 0.0623 & 0.8195\end{array}\right]$} & {$\left[\begin{array}{lll}0.0091 & 0.9239 & 0.0131\end{array}\right]$} \\
\hline 13 & Normal & {$\left[\begin{array}{lll}0.0229 & 0.0080 & 0.9502\end{array}\right]$} & {$\left[\begin{array}{llll}0.0154 & 0.0122 & 0.8070\end{array}\right]$} & {$\left[\begin{array}{lll}0.0009 & 0.9472 & 0.0305\end{array}\right]$} & {$\left[\begin{array}{lll}0.0142 & 0.8605 & 0.0072\end{array}\right]$} \\
\hline 14 & Normal & {$\left[\begin{array}{lll}0.0041 & 0.9917 & 0.0153\end{array}\right]$} & {$\left[\begin{array}{lll}0.0073 & 0.9109 & 0.0251\end{array}\right]$} & {$\left[\begin{array}{lll}0.0100 & 0.8939 & 0.0482\end{array}\right]$} & {$\left[\begin{array}{lll}0.0026 & 0.0174 & 0.9523\end{array}\right]$} \\
\hline 15 & & {$\left[\begin{array}{llll}0.0331 & 0.8074 & 0.0192\end{array}\right]$} & {$\left[\begin{array}{llll}0.0192 & 0.9233 & 0.0095\end{array}\right]$} & {$\left[\begin{array}{lll}0.0226 & 0.9752 & 0.0351\end{array}\right]$} & {$\left[\begin{array}{lll}0.0135 & 0.9802 & 0.0402\end{array}\right]$} \\
\hline 16 & Normal & {$\left[\begin{array}{lll}0.0220 & 0.9455 & 0.0107\end{array}\right]$} & {$\left[\begin{array}{lll}0.0360 & 0.9520 & 0.0142\end{array}\right.$} & {$\left[\begin{array}{lll}0.0042 & 0.9110 & 0.0025\end{array}\right.$} & {$\left[\begin{array}{lll}0.0560 & 0.9785 & 0.0311\end{array}\right]$} \\
\hline 17 & Severe & {$\left[\begin{array}{lll}0.0186 & 0.0233 & 0.9420\end{array}\right]$} & {$\left[\begin{array}{llll}0.0102 & 0.0172 & 0.9611\end{array}\right]$} & {$\left[\begin{array}{llll}0.0611 & 0.0009 & 0.9818\end{array}\right]$} & {$\left[\begin{array}{lll}0.0031 & 0.0576 & 0.9008\end{array}\right]$} \\
\hline 18 & Severe & {$\left[\begin{array}{lll}0.0315 & 0.0203 & 0.8591\end{array}\right]$} & {$\left[\begin{array}{lll}0.0360 & 0.0283 & 0.9838\end{array}\right]$} & {$\left[\begin{array}{lll}0.0223 & 0.0349 & 0.8841\end{array}\right]$} & {$\left[\begin{array}{lll}0.0510 & 0.0448 & 0.9177\end{array}\right]$} \\
\hline 19 & Severe & {$\left[\begin{array}{lll}0.0140 & 0.0113 & 0.9302\end{array}\right]$} & {$\left[\begin{array}{llll}0.0109 & 0.0086 & 0.9779\end{array}\right]$} & {$\left[\begin{array}{lll}0.0075 & 0.0570 & 0.9308\end{array}\right]$} & {$\left[\begin{array}{lll}0.0195 & 0.0473 & 0.9220\end{array}\right]$} \\
\hline 20 & Severe & {$\left[\begin{array}{lll}0.0500 & 0.0396 & 0.9729\end{array}\right]$} & {$\left[\begin{array}{llll}0.0077 & 0.0428 & 0.9601\end{array}\right]$} & {$\left[\begin{array}{lll}0.0197 & 0.0411 & 0.9195\end{array}\right]$} & {$\left[\begin{array}{lll}0.0080 & 0.0166 & 0.8753\end{array}\right]$} \\
\hline 21 & Severe & {$\left[\begin{array}{lll}0.0107 & 0.0133 & 0.8017\end{array}\right]$} & {$\left[\begin{array}{lll}0.0145 & 0.0167 & 0.9810\end{array}\right]$} & {$\left[\begin{array}{lll}0.0083 & 0.0049 & 0.9001\end{array}\right]$} & {$\left[\begin{array}{lll}0.0311 & 0.0150 & 0.9741\end{array}\right]$} \\
\hline 22 & Severe & {$\left[\begin{array}{lll}0.0371 & 0.0091 & 0.9152\end{array}\right]$} & {$\left[\begin{array}{llll}0.0013 & 0.0085 & 0.9077\end{array}\right]$} & {$\left[\begin{array}{llll}0.0073 & 0.0530 & 0.8795\end{array}\right]$} & {$\left[\begin{array}{lll}0.0241 & 0.0318 & 0.9109\end{array}\right.$} \\
\hline 23 & Severe & {$\left[\begin{array}{lll}0.0960 & 0.0531 & 0.9138\end{array}\right]$} & {$\left[\begin{array}{llll}0.0161 & 0.9403 & 0.0544\end{array}\right]$} & {$\left[\begin{array}{lll}0.0106 & 0.0331 & 0.9544\end{array}\right]$} & {$\left[\begin{array}{lll}0.0622 & 0.0373 & 0.9625\end{array}\right]$} \\
\hline 24 & Severe & {$\left[\begin{array}{lll}0.0357 & 0.0581 & 0.7940\end{array}\right]$} & {$\left[\begin{array}{lll}0.0209 & 0.0101 & 0.9632\end{array}\right]$} & {$\left[\begin{array}{lll}0.0096 & 0.0088 & 0.9417\end{array}\right]$} & {$\left[\begin{array}{lll}0.0420 & 0.0307 & 0.9600\end{array}\right]$} \\
\hline
\end{tabular}

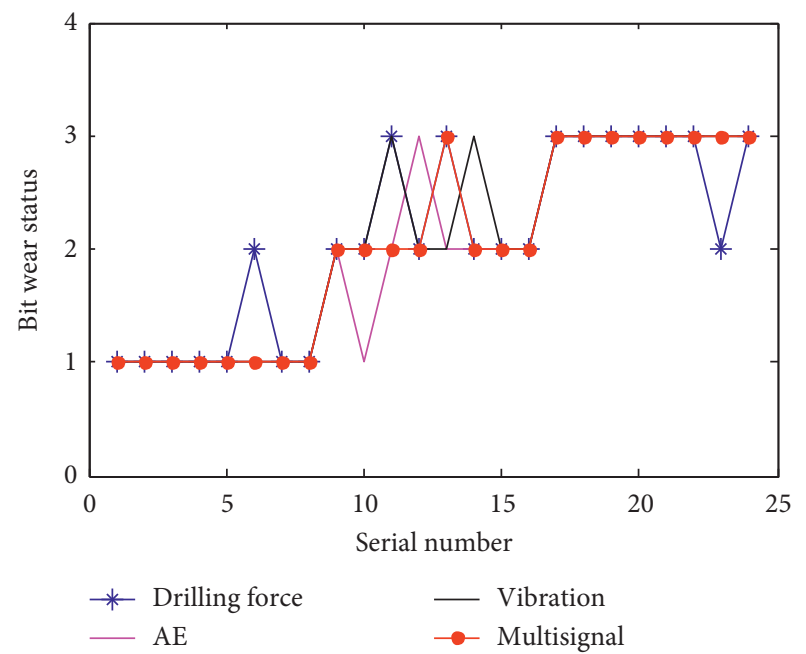

FIGURE 10: Recognition degree of bit wear.

training sample set, and the number of neurons in the output layer is determined by the number of categories that need to be divided in the bit wear state. According to the characteristic frequency band of drilling force, acoustic emission, and vibration signal extracted by wavelet decomposition, the feature vectors of the network learning samples selected in this paper are 8 , so the number of neurons in the input layer of BP neural network is determined to be 8 . The wear state of a bit is divided into three states to be identified: initial wear, normal wear, and severe wear. Therefore, the number of output layer neurons of the BP neural network is determined as 3 .
According to Kolmogorov's theorem, the number of neurons in the hidden layer is as follows:

$$
l=2 \times N+1,
$$

where $L$ is the number of neurons in the hidden layer. $N$ is the number of neurons in the input layer.

According to the eigenvector $N=8$, the number of neurons in the hidden layer is $l=2 \times 8+1=17$.

4.1.3. Determination of Transfer Function. Since the transfer function of the BP neural network must be continuous and differentiable, the sigmoid type function is often used [19]. In this paper, the logarithm S-function (logit) is selected as the output layer transfer function of the BP neural network. It limits the output of the whole network to $[0,1]$, which is close to the output target of the network, so it is convenient to judge the result. To avoid the repetition of the transfer function, the tangent S-type function (Tansig) is selected as the transfer function in the hidden layer, which has the characteristics of saturation and good fault tolerance.

In conclusion, the model used to identify the bit wear state in this paper is a three-layer BP neural network with 817-3 neurons in the input layer, 17 neurons in the hidden layer, and 3 neurons in the output layer. Its structure is shown in Figure 8. The transfer function in the hidden layer is a tangent $S$-type function, and the transfer function in the output layer is a logarithmic $S$-type function.

In Figure 8 , the input layers $x_{1}-x_{8}$ are, respectively, the wavelet energy coefficients of D4 and A5 frequency bands of the drilling force signal after wavelet decomposition, the wavelet energy coefficients of D4, D1, and A5 frequency bands 
TABLE 6: The influence of different network structures and neurons on the recognition results.

\begin{tabular}{|c|c|c|c|c|c|c|c|c|}
\hline $\begin{array}{l}\text { Network } \\
\text { layers }\end{array}$ & $\begin{array}{c}\text { Number } \\
\text { neurons } \\
\text { (single } \\
\text { signal) }\end{array}$ & & $\begin{array}{l}\text { Recognition } \\
\text { rate }\end{array}$ & $\begin{array}{c}\text { Number of neurons (fusion } \\
\text { two signals) }\end{array}$ & & $\begin{array}{l}\text { Recognition } \\
\text { rate }\end{array}$ & $\begin{array}{l}\text { Number of neurons (fusion } \\
\text { of three signals) }\end{array}$ & $\begin{array}{l}\text { Recognition } \\
\text { rate }\end{array}$ \\
\hline \multirow{3}{*}{2} & Force & 2 & 0.7917 & $\begin{array}{c}\text { Fusion of force signal and } \\
\text { AE signal }\end{array}$ & 5 & 0.8333 & \multirow{3}{*}{8} & \multirow{3}{*}{0.9166} \\
\hline & $\mathrm{AE}$ & 3 & 0.8333 & $\begin{array}{c}\text { Fusion of force signal and } \\
\text { vibration signal }\end{array}$ & 5 & 0.7917 & & \\
\hline & Vibration & 3 & 0.8750 & $\begin{array}{c}\text { Fusion of AE signal and } \\
\text { vibration signal }\end{array}$ & 6 & 0.9166 & & \\
\hline \multirow{3}{*}{3} & Force & 2 & 0.8333 & $\begin{array}{c}\text { Fusion of force signal and } \\
\text { AE signal }\end{array}$ & 5 & 0.7917 & \multirow{3}{*}{8} & \multirow{3}{*}{0.9583} \\
\hline & $\mathrm{AE}$ & 3 & 0.9166 & $\begin{array}{c}\text { Fusion of force signal and } \\
\text { vibration signal }\end{array}$ & 5 & 0.916 & & \\
\hline & Vibration & 3 & 0.9166 & $\begin{array}{c}\text { Fusion of AE signal and } \\
\text { vibration signal }\end{array}$ & 6 & 0.9583 & & \\
\hline \multirow{3}{*}{4} & Force & 2 & 0.8750 & $\begin{array}{c}\text { Fusion of force signal and } \\
\text { AE signal }\end{array}$ & 5 & 0.9166 & \multirow{3}{*}{8} & \multirow{3}{*}{1.0000} \\
\hline & $\mathrm{AE}$ & 3 & 0.9583 & $\begin{array}{c}\text { Fusion of force signal and } \\
\text { vibration signal }\end{array}$ & 5 & 0.9583 & & \\
\hline & Vibration & 3 & 0.9166 & $\begin{array}{l}\text { Fusion of AE signal and } \\
\text { vibration signal }\end{array}$ & 6 & 0.9583 & & \\
\hline \multirow{3}{*}{5} & Force & 2 & 0.8750 & $\begin{array}{c}\text { Fusion of force signal and } \\
\text { AE signal }\end{array}$ & 5 & 0.8333 & \multirow{3}{*}{8} & \multirow{3}{*}{0.9166} \\
\hline & $\mathrm{AE}$ & 3 & 0.8750 & $\begin{array}{c}\text { Fusion of force signal and } \\
\text { vibration signal }\end{array}$ & 5 & 0.9166 & & \\
\hline & Vibration & 3 & 0.9166 & $\begin{array}{l}\text { Fusion of AE signal and } \\
\text { vibration signal }\end{array}$ & 6 & 0.9583 & & \\
\hline
\end{tabular}

of the vibration signal, and the wavelet energy coefficients of D1, D2, and D3 frequency bands of AE signal. The output layer $y_{1}$ is $\left[\begin{array}{lll}1 & 0 & 0\end{array}\right] ; y_{2}$ is $\left[\begin{array}{lll}0 & 1 & 0\end{array}\right] ; y_{3}$ is $\left[\begin{array}{lll}0 & 0 & 1\end{array}\right]$, and the corresponding relationship with the bit wear status is shown in Table 4.

4.2. Training of Neural Network. In this paper, 15 sets of data of drill bit in three states of initial wear, normal wear, and severe wear are selected, and 45 sets of data are taken as training samples. The data are normalized by premnmx function, which limits the value of sample data to the range of $[-1,1]$, so that the network can quickly collect and improve the stability of the network. The call form of premnmx function is as follows [20]:

$[p n, \min p, \max p, t n, \min t, \max t]=\operatorname{premnmx}(p, t)$.

The normalized vector is regarded as the input of BP neural network, and the bit where state is regarded as the expected output. The training error of the network is 0.0001 , and the learning rate is 0.1 . The training error curve is shown in Figure 9, and the neural network model reaches the target error after 60 times of training.

4.3. Comparative Analysis of Test Results. To compare the recognition results of multisignal fusion and single signal monitoring bit wear state, a comparative test of drilling force signal and multisignal fusion monitoring bit wear state is carried out, and the recognition results of drilling force signal and multisignal fusion are compared and analyzed.
In the nontraining book, there are 10 groups of initial wear, 16 groups of normal wear, and 9 groups of serious wear in a total of 35 groups of data; 8 groups of data in each of the three states are randomly selected as test samples, which are input into the established neural network model for testing, and the comparison results of single signal and multisignal fusion recognition of bit wear state are shown in Table 5.

To facilitate analysis and comparison, normalize the test results and draw a comparison diagram as shown in Figure 10.

In Figure 10, the abscissa is the data serial number. The actual bit state in serial number $1-8$ is the initial wear, normal wear in serial number 9-16, and severe wear in serial number 17-24. The ordinate is the model identification result, in which 1, 2, and 3 represent the initial stage, normal, and severe wear stage of the bit, respectively. When a single signal of drilling force is used to monitor bit wear, one initial wear (Group 6 data) is misjudged as normal wear, two normal wears (groups 11 and 13 data) are misjudged as serious wear, one serious wear (Group 23 data) is misjudged as normal wear, and the accuracy of bit wear is $83.3 \%$; vibration single signal is used to monitor bit wear. In the state of acoustic emission, two normal wears (Group 11 and group 14 data) are misjudged as serious wear, and the accuracy rate of bit wear state discrimination is $91.6 \%$; in the state of monitoring bit wear with single acoustic emission signal, one normal wear (Group 10 data) is misjudged as initial wear and one normal wear (Group 12 data) is misjudged as serious wear, and in the state of bit wear 
discrimination, one normal wear (Group 12 data) is misjudged as serious wear. The accuracy rate is $91.6 \%$; a normal wear (the 13th group of data) is misjudged as serious wear by the combination of drilling force, AE, and vibration signals. The accuracy rate of bit wear state recognition is $95.8 \%$, which improves the accuracy rate of bit wear state recognition.

4.4. Structure Optimization of Neural Network Model. To reduce the error of neural network model in tool condition monitoring, this paper optimizes the structure of neural network model, takes the above training samples as input data, analyzes the influence of network layer and neuron number on the recognition effect, and the analysis results are shown in Table 6. It can be seen from Table 6 that with the increase of the number of layers and neurons in the network, the recognition rate of tool state does not always increase. This is because the signal is nonstationary and the recognition result is interfered by the outside world. When the signal is fused under different network structures, it may cause interference among different signals. Among them, when the number of layers in the network is 4 , the force signal, acoustic emission signal, and vibration signal, The highest accuracy of No. 1 fusion is $100 \%$. The neural network structure model is optimized reasonably.

\section{Conclusion}

In this paper, a multisignal acquisition system based on drilling force, vibration, and AE signals is set up to monitor the wear state of 7075 aluminum plates on a $40 \mathrm{kHz}$ ultrasonic vibration drilling device, and the results are compared with those of the single signal of drilling force. The conclusion is as follows:

(1) Based on the improved LMD method of noise, the wavelet energy of the axial drilling force signal is most obvious in bands D4 and A5; the wavelet energy of vibration signal in bands D1, D4, and A5 fluctuates greatly, and the wavelet energy of $\mathrm{AE}$ signal is most obvious in bands D1, D2, and D3. Therefore, the above eight eigenvalues can be used as the characteristic parameters to identify the tool wear state.

(2) A 3-layer BP neural network model of 8-17-3 is established to identify the wear state of the drill bit. The above 8 eigenvalues are input into the recognition model for training and testing. After 60 times of training, the network model achieves the target error of 0.0001 .

(3) A comparative test of single signal and multisignal fusion monitoring bit wear state is carried out. The results show that the accuracy of discrimination is $83.3 \%, 91.6 \%, 91.6 \%$, and $95.8 \%$, respectively, when the single signal of drilling force is used to monitor the wear state of the bit, vibration is used to monitor the wear state of the bit, acoustic emission is used to monitor the wear state of the bit, and multisignal fusion is used to identify the wear state of bit. Multisignal fusion method is used to improve the accuracy of bit wear state recognition.

(4) Using four layers of network layer, the accuracy rate of force signal, acoustic emission signal, and vibration signal fusion monitoring vibration drilling tool state is up to $100 \%$, and the neural network structure model is optimized reasonably.

\section{Data Availability}

The experimental data access address applied in this paper is https://pan.baidu.com/s/1xrILwP7umf3MQhKfzk6w (keys: 4w8s.P9).

\section{Conflicts of Interest}

The authors declare that they have no conflicts of interest.

\section{Acknowledgments}

This project was funded by China's Ministry of Education Chunhui Project (2018JB301L08), Jilin Provincial Development and Reform Commission (2019C040-1), and Changchun Science and Technology Bureau (18DY031).

\section{References}

[1] S. Chen, P. Zou, H. Wu, D. Kang, and W. Wang, "Mechanism of chip formation in ultrasonic vibration drilling and experimental research," Proceedings of the Institution of Mechanical Engineers, Part C: Journal of Mechanical Engineering Science, vol. 233, no. 15, pp. 5214-5226, 2019.

[2] D. R. Salgado and F. J. Alonso, "Tool wear detection in turning operation using singular spectrum analysis," Journal of Materials Processing, vol. 22, pp. 735-748, 2008.

[3] S. K. Choudhury and S. Rath, "In-process tool wear estimation in milling using cutting force model," Journal of Materials Processing Technology, vol. 99, no. 1-3, pp. 113-119, 2000.

[4] K. T. Chung and A. Geddam, "A multi-sensor approach to the monitoring of end milling operations," Journal of Materials Processing Technology, vol. 139, no. 1, pp. 15-20, 2003.

[5] F. A. Al-Sulaiman, M. A. Baseer, and A. K. Sheikh, "Use of electrical power for online monitoring of tool condition," Journal of Materials Processing Technology, vol. 166, no. 3, pp. 364-371, 2005.

[6] S. K. Sikdar and M. Chen, "Relationship between tool flank wear area and component forces in single point turing," Journal of Materials Processing Technology, vol. 128, no. 1-3, pp. 210-215, 2002.

[7] H. Li, H. Kan, Z. Wei et al., "Investigation on milling cutter monitoring in the process of complex surface machining," Journal of Vibration, Menoasurement \& Diagnosis, vol. 38, no. 04, pp. 658-665, 2018.

[8] L. Hongkun, H. A. O. Baitian, D. Yuebang, and Y. Rui, "Wear status recognition for milling cutter based on compressed sensing and noise stacking sparse auto-encoder," Journal of Mechanical Engineering, vol. 55, no. 14, 2019.

[9] A. I. Azmi, "Monitoring of tool wear using measured machining forces and neuro-fuzzy modelling approaches during 
machining of GFRP composites," Advances in Engineering Software, vol. 82, pp. 53-64, 2015.

[10] W. Gao, Research on Tool Wear of Deep Hole Machining and State Recognition, North University of China, Taiyuan, China, 2017.

[11] J. S. Smith, "The local mean decomposition and its application to EEG perception data," Journal of the Royal Society Interface, vol. 2, no. 5, pp. 444-450, 2005.

[12] B. Zhang and C. Zhao, "Dynamic turning force prediction and feature parameters extraction of machine tool based on ARMA and HHT," Proceedings of the Institution of Mechanical Engineers, Part C: Journal of Mechanical Engineering Science, vol. 234, no. 5, pp. 1044-1056, 2020.

[13] K. Wang, Q. Hao, X. Zhang et al., Blind Source Extraction of Acoustic Emission Signals for Rail Cracks Based on Ensemble Empirical Mode Decomposition and Constrained Independent Component Analysis, Elsevier Ltd., Amsterdam, Netherlands, 2020.

[14] P. Tiwari and S. H. Upadhyay, "Elucidation of ball bearing performance utilizing product functions of vibration signals and locality sensitive discriminant analysis," Proceedings of the Institution of Mechanical Engineers, vol. 234, no. 1, pp. 50-66, 2020.

[15] X.-B. Fan, B. Zhao, and B.-X. Fan, "Wavelet decomposition and nonlinear prediction of nonstationary vibration signals," Noise \& Vibration Worldwide, vol. 51, no. 3, pp. 52-59, 2020.

[16] X. Ku, B. Cao, Y. Guo et al., "A data processing method for tool wear monitoring," Machine Tool \& Hydraulics, vol. 45, no. 17, pp. 105-109, 2017.

[17] Y. Li, J. Li, J. Huang et al., "Fitting analysis and research of measured data of SAW micro-pressure sensor based on BP neural network," Measurement, vol. 155, 2020.

[18] V. Kurková, "Kolmogorov's theorem and multilayer neural networks," Neural Networks, vol. 5, no. 3, pp. 501-506, 1992.

[19] J. Chen, Z. Liang, G. Zhang et al., "Applying ANN and BP algorithm to identify static model construction of the directfired LiBr absorption chiller," Journal of Hunan University (Natural Sciences), vol. 11, no. S3, pp. 60-66, 2000.

[20] L. Jin-Bo, -X. Xin, Y. W. Yue, and C. K. Wei, "An on-line method for multi-license plates recognition based on neural network and support vector machine," Acta Automatica Sinica, vol. 1-10, 2019. 